\title{
Réponse d'une turbomachine hydraulique à des luctuations des paramètres dynamiques du circuit
}

\author{
Response of a hydraulic turbomachine to fluctuation \\ of dynamic circuit parameters
}

Michele Fanelli" et Framco Siccardi ${ }^{*}$ *

Une turbomachine insérée dans un circuit hydraulique influe d'une manière particulièrement complexe sur les perturbations (périodiques ou aléatoires) $\mathrm{du}$ régime hydraulique du circuit.

En effet, chaque machine est en premier lieu une source (ou générateur) de perturbations, qui excitent le reste du circuit (rôle actif).

Deuxièmement, chaque machine impose des conditions aux limites particulièrement compliquées aux perturbations se propageant dans les autres éléments du circuit (rôle passif).

Si l'on considère, de plus, que des effets non-linéaires sont sûrement en jeu, et que les perturbations en question peuvent survenir autour de l'un quelconque des nombreux points de fonctionnement à régime possible en général pour une machine, ou encore pendant les évolutions hors de régime (c'est-à-dire en "transitoire"), on peut envisager l'impossibilité pratique de prévoir l'allure quantitative et même qualitative des phénomènes en question.

En effet, les études du comportement non-stationnaire des turbomachines sont extrêmement difficiles, qu'il s'agisse d'un modèle analy tique ou numérique ou bien de relevés expérimentaux; ce qui fait que le sujet n'ait été abordé que depuis des temps relativement récents et par un nombre relativement réduit de chercheurs.

D'autre part, l'intérêt pour une telle question est bien loin d'être purement académique.

En effet, on assiste au développement de turbomachines de plus en plus puissantes et de grandes dimen-

(*) Chef du Service d'Analyses Théoriques du Centre de Recherches d'Hydraulique et de Structures de la Direction d'Etudes et Recherches d'ENEL.

(**) Professeur à l'Institut d'Hydraulique de l'Ecole d'Ingénieurs de l'Université de Gênes. sions, ce qui conduit d'une part à la dissipation d'une puissance considérable (en valeur absolue, sinon en proportion relative), avec étalement de cette puissance sur un certain spectre de fréquences ; d'autre part à la réalisation de pièces mécaniques de plus en plus minces, donc flexibles, donc capables d'intéragir, par hydroélasticité, avec les perturbations hydrauliques, et d'entretenir ou d'exciter des niveaux de vibration dangereux.

De plus, dans les installations modernes d'accumulation d'énergie hydraulique par pompage (installations qui se présentent comme le complément rationnel des centrales de production de base, que ce soient des centrales à fuel ou des centrales nucléaires) les exigences de service conduisent à avoir plusieurs mancuvres d'arrêt ou de démarrage par jour : donc les occasions pendant lesquelles on traverse des plages éloignées du fonctionnement optimal du groupe (et du circuit), en risquant ainsi l'excitation de conditions perturbées, peuvent survenir avec une cadence élévée. D'où la possibilité d'une accumulation de dommages mécaniques par fatigue même dans les pièces qui ne seraient sollicitées, par les perturbations de signe alterné, qu’à un taux de contraintes relativement modeste.

\section{Le modèle linéaire}

Faute de mieux, on suppose en général qu'il soit admissible de rester dans l'approximation des perturbations d'amplitude réduite (à la limite, d'amplitude infinitésimale) et des effets superposables (phénomènes linéaires).

On peut alors caractériser en principe le comportement de la machine par une "matrice de transfert" reliant entre elles un certain nombre de grandeurs 
pulsatoires d'entrée et de sortie, soit :

$q_{e} \quad$ débit pulsatoire d'entrée

$q_{s}$ débit pulsatoire de sortie ;

$h_{e} \quad$ variation pulsatoire de la charge piézométrique à l'entrée :

$h_{s} \quad$ variation pulsatoire de la charge piézométrique à la sortie ;

$c_{r} \quad$ variation pulsatoire $\mathrm{du}$ couple hydraulique sur la roue ;

$\Omega \quad$ variation pulsatoire de la vitesse angulaire de la roue ;

$\nu \quad$ variation pulsatoire de l'ouverture du distributeur, pour les machines à distributeur mobile.

(Ces dernières variations étant en général dues aux variations périodiques dans le circuit de régulation, par l'intermédiaire des variations $\Omega$, augmentées le cas échéant des variations dues à la déformation élastique périodique des aubes du. distributeur, et de toute la chaîne cinématique leur afférant, sous l'effet des charges hydrauliques pulsatoires).

S'il y a une ligne d'arbre longue et flexible (pour ce qui est des oscillations de torsion), il y aura lieu de distinguer entre les variations :

$\Omega$ de la vitesse angulaire de la roue hydraulique, $\Omega_{e}$ de la vitesse angulaire de la machine électrique.

Afin de comparer les résultats sur différentes machines on peut mettre sous forme non-dimensionnelle les grandeurs pulsatoires. On a :

$$
\varphi_{e, s}=q_{e, s} /\left(\Omega_{o} D^{3}\right)
$$

pour les coefficients de débit

et

$$
\psi_{e, s}=g h_{e, s} /\left(\Omega_{o}^{2} D^{2}\right)
$$

pour les coefficients de pression

On a aussi

$$
\tau=c_{r} /\left(\rho \Omega_{o}^{2} D^{5}\right)
$$

pour le coefficient du couple

et

$$
\nu=\Omega / \Omega_{o}
$$

pour les pulsations réduites de la vitesse angulaire de la roue,

où l'on a indiqué par $\Omega_{o}$ la vitesse angulaire moyenne de la roue, $D$ son diamètre caractéristique et $\rho$ la densité moyenne du fluide.

Le diagramme en blocs correspondant au schéma cidessus est représenté fig. 1 .

\section{Comportement passif}

Il est bien connu, d'après la théorie des systèmes linéaires, que si l'on est capable de décrire le comportement de la machine (les relations existant entre les différentes grandeurs ci-dessus) en regime sinusoïdal pur, en fonction de la pulsation $\omega(\omega=2 \pi f ; f=$ fréquence

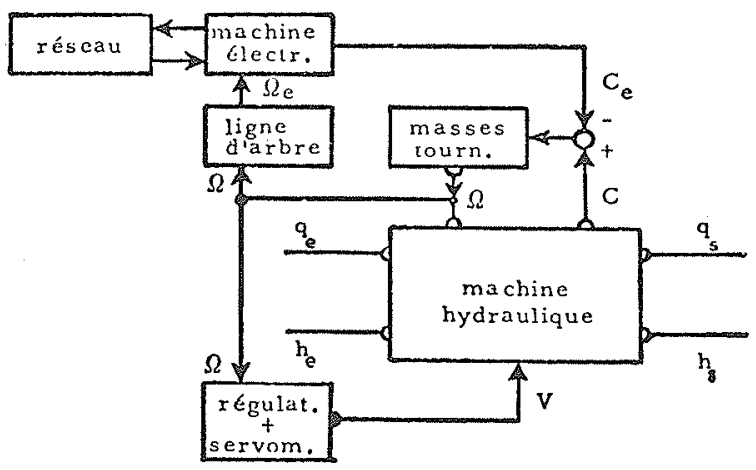

Figure 1 - Diagramme en blocs du schéma de la machine.

en cycles/s ; $f=1 / T$ avec $T=$ période), alors en principe on peut calculer la réponse de la machine en régime transitoire quelconque.

Ces relations, ou fonctions de transfert, peuvent être groupées d'une façon très efficace dans une matrice de transfert : par exemple, en supposant que les aubes du distributeur soient fixes (pompe) ou bloquées, on pourra écrire :

$\left\{\begin{array}{c}h_{s}-h_{e} \\ c_{r} \\ q_{s}-q_{e}\end{array}\right\}=\left[\begin{array}{ccc}\eta_{h}^{*} & \eta_{\Omega}^{*} & \eta_{q}^{*} \\ \mu_{h}^{*} & \mu_{\Omega}^{*} & \mu_{q}^{*} \\ \chi_{h}^{*} & \chi_{\Omega}^{*} & \chi_{q}^{*}\end{array}\right] \cdot\left\{\begin{array}{l}h_{e} \\ \Omega \\ q_{e}\end{array}\right\}=\left[T^{*}\right] \cdot\left\{\begin{array}{c}h_{e} \\ \Omega \\ q_{e}\end{array}\right\}$

ou bien, sous forme non-dimensionnelle,

$$
\left\{\begin{array}{c}
\psi_{s}-\psi_{e} \\
\tau \\
\varphi_{s}-\varphi_{e}
\end{array}\right\}=\left[\begin{array}{ccc}
\eta_{h} & \eta_{\Omega} & \eta_{q} \\
\mu_{h} & \mu_{\Omega} & \mu_{q} \\
\chi_{h} & \chi_{\Omega} & \chi_{q}
\end{array}\right] \cdot\left\{\begin{array}{c}
\psi_{e} \\
v \\
\varphi_{e}
\end{array}\right\}=[T] \cdot\left\{\begin{array}{l}
\psi_{e} \\
v \\
\varphi_{e}
\end{array}\right\}
$$

Ici $\left[T^{*}\right]$ est justement la matrice de transfert et $[T]$ la forme nondimensionnelle. On doit retenir que les éléments $\eta_{\Omega} \ldots \chi_{q}$ de $[T]$ sont des fonctions complexes du paramètre (réel positif) $\sigma=\omega / \Omega_{0}$ qui représente la pulsation réduite des perturbations.

En particulier on aura :

$$
\begin{gathered}
\eta_{h}=\frac{\partial\left(\psi_{s}-\psi_{e}\right)}{\partial \psi_{e}}=\frac{\partial\left(h_{s}-h_{e}\right)}{\partial h_{e}}=\eta_{h}(\sigma) \\
\eta_{\Omega}=\frac{\partial\left(\psi_{s}-\psi_{e}\right)}{\partial \nu}=\frac{\partial\left(h_{s}-h_{e}\right)}{\partial \Omega}\left(\Omega_{o} D^{2} / g\right)=\eta_{\Omega}(\sigma) \\
\chi_{q}=\frac{\partial\left(\varphi_{s}-\varphi_{e}\right)}{\partial \varphi_{e}}=\frac{\partial\left(q_{s}-q_{e}\right)}{\partial q_{e}}=\chi_{q}(\sigma)
\end{gathered}
$$

Si l'on accepte certaines hypothèses simplificatrices, quelques-unes de ces fonctions seront nulles. Par exemple, si les parois de la machine sont rigides, le fluide est supposé incompressible et on n'a pas de cavitation $\varphi_{s}=\varphi_{e}$ et par conséquent $\chi_{h}=\chi_{\Omega}=\chi_{q}=0$; aussi $\partial\left(\psi_{s}-\psi_{e}\right) / \partial \psi_{e}=0 ; \partial \tau / \partial \psi_{e}=0$ d'où $\eta_{h}=\mu_{h}=0$.

De plus, ces fonctions doivent évidemment tendre, pour $\sigma \rightarrow 0$, aux valeurs "statiques" qu'on peut tirer des "courbes caractéristiques" de la machine :

$$
\eta_{q} \underset{\sigma \rightarrow 0}{\longrightarrow} \frac{\partial H}{\partial Q} \cdot\left(g D / \Omega_{o}\right) \quad H(Q)
$$


étant la courbe caractéristique de la charge hydraulique en fonction du débit, à vitesse angulaire constante ; courbe caractéristiques établie, comme d'ordinaire, par détermination de plusieurs points en conditions de régime autant que possible permanent.

De même :

$$
\eta_{\Omega} \underset{\sigma \rightarrow 0}{\longrightarrow} \frac{\partial H}{\partial \Omega}\left(g / \Omega_{o} D^{2}\right)
$$

la dérivée partielle étant faite en ce cas à débit constant, sur les mêmes courbes caractéristiques ci-dessus.

En principe, pour déterminer les coefficients de la matrice de transfert $[T]$ introduite auparavant, on pourrait avoir recours à l'une ou à l'autre des deux approches suivantes :

a) analyse théorique ou numérique des variations périodiques du champ d'écoulement à l'intérieur de la roue en régime pulsatoire ;

b) relevés expérimentaux en régime pulsatoire.

En pratique, on se heurte à de très grosses difficultés tant dans le cas a) que dans le cas b).

a) L'analyse (numérique ou théorique) des perturbations harmoniques de l'écoulement à l'intérieur de la roue est non seulement extrêmement difficile en soimême ; au cas où on saurait la faire, elle risquerait d'être inutile vues les incertitudes sur les conditions aux limites appropriées en "amont" et en "aval" de la roue. Par exemple : comment les perturbations réagissent sur l'aspirateur d'une part (composante rotationnelle dans l'aspirateur, en particulier), sur la bâche spirale d'autre part (est-il admissible d'avoir des perturbations de pression tout-à-fait synchrones le long de la bâche ? Ou bien y aura-t-il en général des différences de phase de point à point ?). Autrement dit : est-il possible de définir une matrice de transfert de la machine indépendamment des réactions des composants hydrauliques qui lui sont couplés dans le circuit ?

b) Les relevés expérimentaux devraient être réalisés en conditions, en nombre et avec une précision suffisants à la détermination des coefficients de la matrice de transfert $[T]$; et ceci pour autant de points de fonctionnement "moyens" autour desquels on peut envisager l'établissement d'un régime pulsatoire, et (pour chacun de ces points) au moins pour autant de valeurs de $\omega$ qu'on peut estimer être intéressantes vis-à-vis du circuit où la machine est insérée. De plus, les relevés devraient être réalisés, pour chaque détermination de la matrice $[T]$, avec des amplitudes différentes des phénomènes pulsatoires, afin de vérifier le bien fondé de l'hy. pothèse de linéarité.

c) Ces relevés expérimentaux sont extrêmement délicats, à la suite de plusieurs facteurs défavorables :

$c_{1}$ ) le niveau élevé de "bruit" toujours présent dans le circuit et particulièrement au droit de la machine, d'où un rapport "signal-bruit" souvent très faible, ce qui impose des procédures pénibles et parfois douteuses d'élaboration des mesures;

$c_{2}$ ) il est très difficile, à l'heure actuelle, de mesurer directement certaines des grandeurs variables, et notamment les variations périodiques du débit, $q$;

$c_{3}$ ) l'application, de l'extérieur, de perturbations hydrauliques sinusoïdales pures et exactement contrôlées (en phase, amplitude et fréquence) demande certaines précautions et n'est pas sans difficultés ;

$c_{4}$ ) la présence, assez fréquente, de super-harmoniques (ou plus rarement sub-harmoniques) de la fréquence des perturbations imposées par l'extérieur, ce qui d'une part oblige à effectuer des décompositions à la Fourier pour tirer les coefficients de la matrice $[T]$, mais d'autre part peut être interprété comme symptôme de la présence d'effets non-linéaires et donc, à la rigueur, infirmerait le procédé même de décomposition à la Fourier qui, lui, est admissible en régime de superposition linéaire des effets.

Jusqu'à présent on a fort peu d'informations du point de vue soit théorique soit expérimental ; si l'on se passe de quelques études strictement consacrées à des problèmes particuliers, qui ne sont pas encore parus au niveau international, il nous reste à rappeler les recherches du Laboratoire Machines de la Division de Engineering du Caltech et les recherches du Centre de l'ENEL de Milan, en collaboration avec le Laboratoire d'Hydraulique de l'Université de Gênes.

Les travaux des deux groupes portent sur des machines très différentes : Acosta et maintenant Brennen étudient le comportement dynamique des inducteurs à vitesse spécifique très élevée, particulièrement en condition de cavitation avancée. La première tâche des études consista en effet à expliquer les oscillations de la poussée des vecteurs "Saturn", qui entretenaient des pulsations longitudinales permanentes nommées effet "POGO" : ce qui explique la petite dimension et les caractéristiques des machines qui ont été étudiées.

Fanelli et maintenant Fanelli et Siccardi étudient la réponse dynamique de pompes à vitesse spécifique très réduite, sans cavitation ou avec un degré de cavitation très limité : ça vient du secteur industriel sur lequel se penche la recherche, qui est celui des grands aménagements hydroélectriques de refoulement.

Les différents intérêts industriels des études expliquent aussi les différences entre la façon de représenter la matrice de transfert de la machine et la façon par laquelle les deux groupes conduisent les essais. On essaiera de donner dans les points suivants une esquisse unifiée des méthodologies et des résultats.

1 - Comme on l'a signalé plus haut, il y a bien des difficultés à maîtriser des essais qui aient pour but d'évaluer les sept coefficients de la matrice de transfert, qui sont généralement différents de l'unité. On va donc rechercher des comportements où les uns ou les autres de ces coefficients peuvent être connus à priori afin de réduire la complexité des essais : le premier champ est celui de la réponse dynamique en absence de cavitation, où l'on néglige aussi les effets de compressibilité du fluide et de déformabilité des parois de la machine. On a alors que la matrice se réduit à un quadrupôle.

$$
\left\{\begin{array}{c}
\psi_{s}-\psi_{e} \\
\tau
\end{array}\right\}=\left[\begin{array}{cc}
\eta_{\Omega}(\sigma) & \eta_{q}(\sigma) \\
\mu_{\Omega}(\sigma) & \mu_{q}(\sigma)
\end{array}\right] \cdot\left\{\begin{array}{c}
\nu \\
\varphi_{e}
\end{array}\right\}
$$


où $\sigma=\frac{\omega}{\Omega_{0}}, \Omega_{o}$ étant la vitesse de rotation de la roue.

On obtient le même résultat lorsque le moteur électrique à grande inertie interdit toute fluctuation de vitesse angulaire de l'arbre : on a alors encore

$$
\left\{\begin{array}{c}
\psi_{s}-\psi_{e} \\
\varphi_{s}-\varphi_{e}
\end{array}\right\}=\left[\begin{array}{cc}
\eta_{h}(\sigma) & \eta_{q}(\sigma) \\
\chi_{h}(\sigma) & \chi_{q}(\sigma)
\end{array}\right] \cdot\left\{\begin{array}{c}
\psi_{e} \\
\varphi_{e}
\end{array}\right\}
$$

Dans les deux cas on peut réduire le quadrupôle à une simple impédance lorsque les effets d'inertie du moteur sont très grands, dans le premier cas, ou lorsque les effets de déformabilité du fluide et des parois sont très petits dans le deuxième cas :

$$
\psi_{s}-\psi_{e}=\eta_{q} \varphi
$$

2 - Le cas où l'on a un certain nombre de résultats expérimentaux est en effet celui dans lequel la machine se conduit comme une simple impédance.

Les résultats de Fanelli et de Brennen sont représentés sur les figures 2 et 3 . Il faut rappeler que pour les deux machines on a :

$n_{s}=\varphi^{1 / 2} \psi^{-3 / 4}=.75$ et $\left(n_{q}\right)_{\sigma=0}=-7$ pour la pompe centrifuge

et $n_{s}=\varphi^{1 / 2} \psi^{-3 / 4}=3.35$ et $\left(\eta_{q}\right)_{\sigma=0}=-5$ pour l'inducteur axial.

Les résultats ont été représentés en forme nondimensionnelle directement sur la figure 2. Sur la figure 3 ils ont été réduits à la même échelle en divisant par la valeur statique de l'impédance.

A chaque point expérimental est associée la valeur correspondante de la fréquence réduite de la perturbation qui traverse la machine. On observe que l'allure des deux courbes est nettement différente : pour la machine à petite vitesse spécifique on retrouve que la composante résistive de l'impédance diminue à partir des valeurs de la fréquence réduite très proches de $\sigma=0.1$. Lorsqu'on dépasse $\sigma=0.25$ le comportement dynamique est presque complètement changé de résistif en inertial.

Pour la machine à haute vitesse spécifique on retrouve au contraire que le comportement demeure résistif, bien que la composante d'inertie ait tendance à grandir, jusqu'à fréquence réduite $\sigma=0.25$. Cela dépend, dans l'opinion des auteurs, de la complexité et de la longueur des conduits fixes de la machine centrifuge en comparaison avec ceux de la machine axiale.

Dans les deux cas la réponse de la machine aux perturbations qui se propagent dans le circuit change lorsque la fréquence crô̂t, ce qui change les conditions de résonance du circuit d'une façon qui peut être, dès maintenant, connue au moins à un niveau qualitatif.

3 - Lorsque la machine est affectée par un degré de cavitation non négligeable, la réponse dynamique est tout à fait différente de celle qu'on a présentée jusque-là.

La contribution des termes diagonaux de la matrice de transfert n'est plus négligeable. Il apparait donc un effet de gain qui est lié à $\chi_{q}$ et un effet de compressibilité qui est lié à $\chi_{h}$ (dans les pulsations de débit), tandis que dans les pulsations de pression apparait un effet d'amplification lié à $\eta_{h}$.

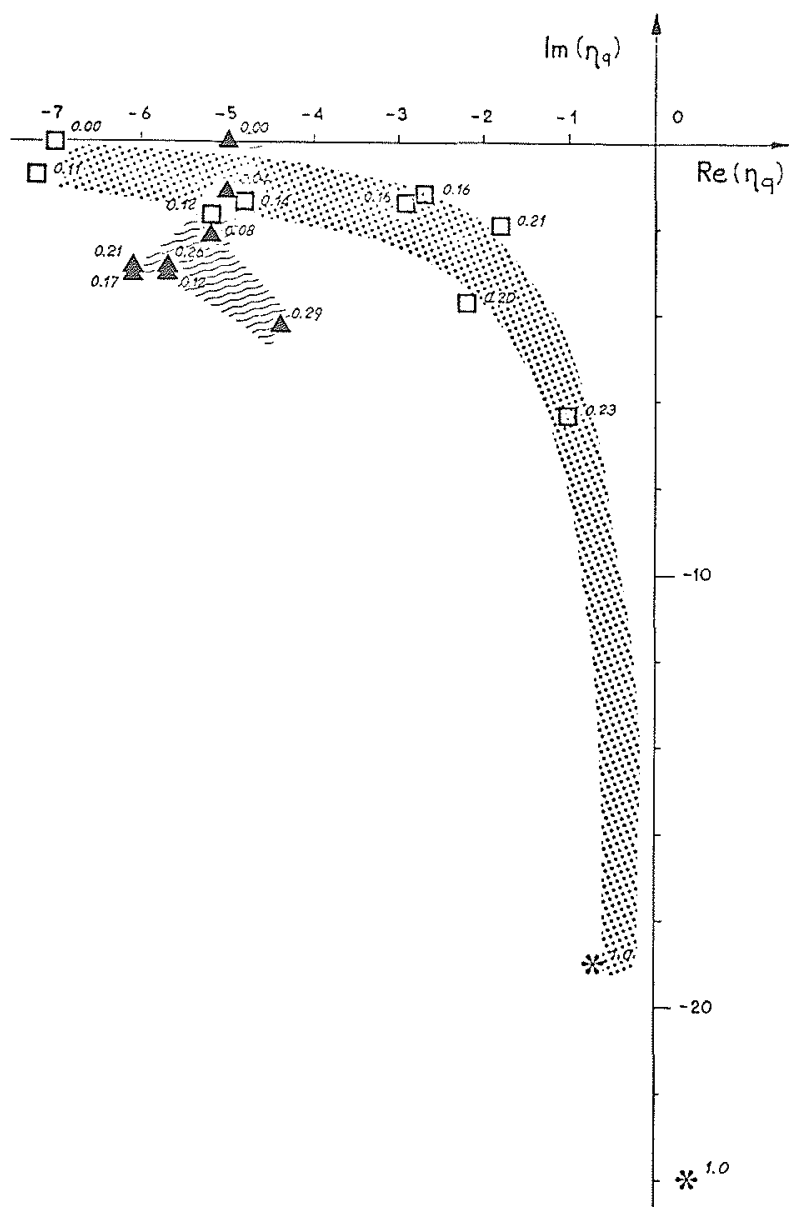

Figure 2 - Comparaison des résultats de Fanelli (ㅁ) (1972), Ng et Brennen (A) (1978) et Siccardi $\left({ }^{*}\right)(1976)$.

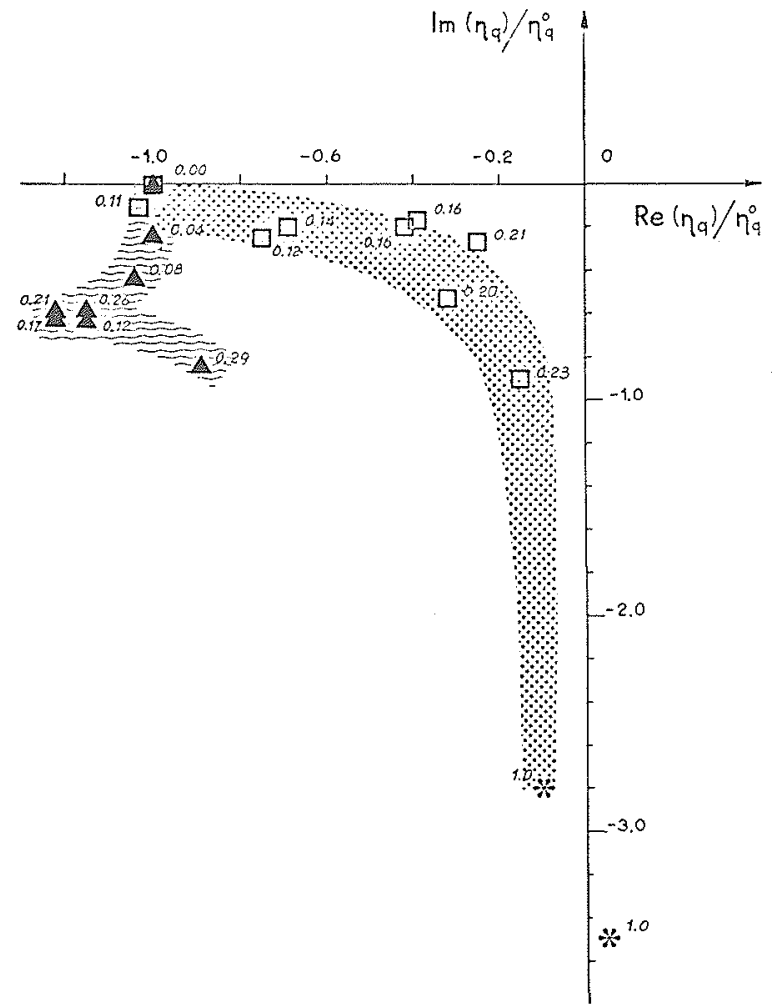

Figure 3 - Comparaison des résultats de Fanelli (a), Ng et Brennen (A) et Siccardi $(*)$ : échelle unifíée. 

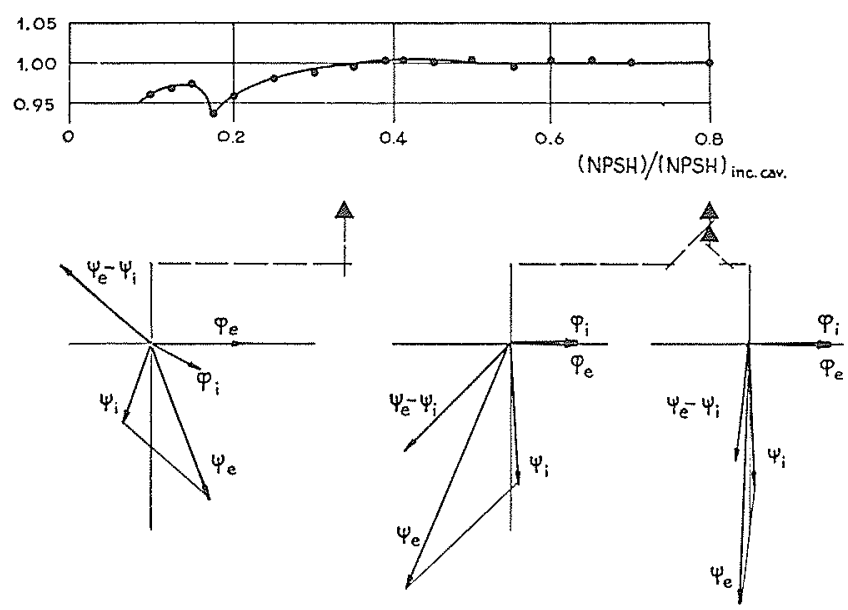

$\left.b_{2}\right)$

$\left.b_{1}\right)$

a)
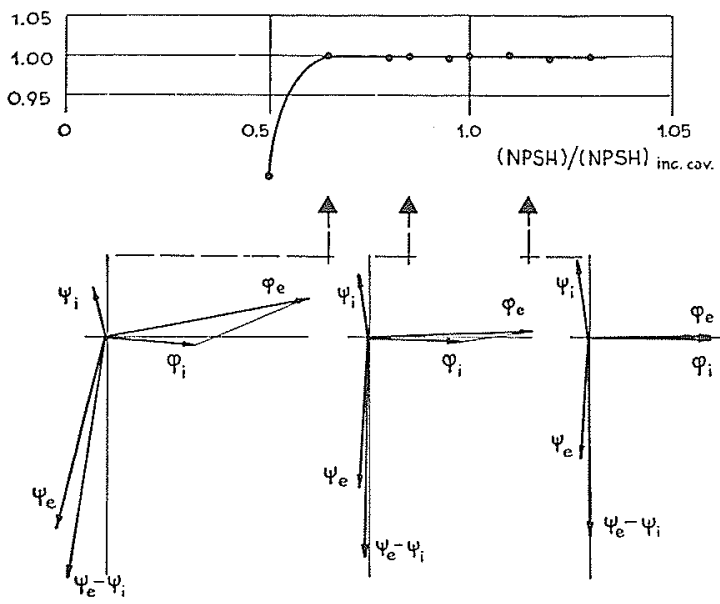

$$
c_{3} \text { ) }
$$

$\left.c_{2}\right)$

$\left.c_{1}\right)$

Figure 4 - Réponse dynamique d'une pompe à des oscillations de débit à la sortie selon le degré de cavitation et la fréquence réduite des perturbations.

a - Inducteur, cavitation modérée, $\sigma=0.01$ (Brennen, 1978) impédance des conduites d'aspiration et de refoulement $\mathrm{du}$ même ordre que celle de l'inducteur $\left(\left|Z_{i}\right| \simeq\left|Z_{e}\right| \simeq\left|\eta_{q}\right|\right)$

$b_{1}$ - Inducteur, cavitation moderée, $\sigma=0.25$ (Brennen, 1978)

$\mathrm{b}_{2}$ - Inducteur, cavitation développée, $\sigma=0.25$ (Brennen, 1978)

$c_{1}$ - Pompe centrifuge, absence de cavitation, pompe comme source de perturbations : $\sigma \simeq 1$ (Siccardi, 1976). Impédance des conduites d'aspiration et de refoulement du même ordre que celle de la pompe $\left(\left|Z_{i}\right| \simeq\left|Z_{e}\right| \simeq\left|\eta_{g}\right|\right)$. Conduite d'aspiration entre la première résonance et la première antirésonance.

$c_{2}$ - Pompe centrifuge, légère cavitation, $\sigma \simeq 1$ (Siccardi, 1976)

$c_{3}$ - Pompe centrifuge, cavitation moderée, $\sigma \simeq 1$ (Siccardi, 1976).

La valeur des effets que le phénomène de cavitation introduit dans le comportement dynamique de la machine a été mesurée par Brennen sur de petits inducteurs de vitesse spécifique élevée. Sur la figure 4 on a schématisé quelques résultats dans les deux cas d'inducteur sans et avec cavitation à l'entrée : il faut d'abord s'apercevoir que les effets sont du même ordre des effets semblables, qui sont introduits par la déformabilité et l'inertie des composants du circuit.

L'influence de la cavitation est toutefois moins sensible sur machines à haute vitesse spécifique que sur machines à faible vitesse spécifique. Pour apprécier un gain de débit ou une amplification des pressions importants on doit atteindre sur les inducteurs des extensions des poches de cavitation, qui occupent presque la première hélice des aubes. Au contraire, comme on peut le voir encore sur la figure 4 , sur des machines centrifuges l'effet des volumes cavitants est évident lorsque le point de fonctionnement de la machine est encore bien au-dessus de la crise de cavitation.

$\mathrm{Si}$ la détermination théorique de la réponse de la machine en l'absence de cavitation a quelque peu progressé, pour le cas de cavitation on a seulement recours à des modèles semi-empiriques qui permettent une interprétation qualitative des allures expérimentales ${ }^{\left({ }^{1}\right.}$.

Il faut toutefois observer que la cavitation change complètement la réponse dynamique, car elle introduit, à la place d'un composant résistif-inertial, un composant capacitif très net : cela peut provoquer, voir par exemple l'effet "POGO" déjà cité, des résonances en basse fréquence dans la partie aspiration des circuits.

4 - On a encore à examiner le deuxième schéma possible de quadrupôle, que l'on obtient lorsque les effets de cavitation sont négligeables mais l'inertie du moteur n'est pas si grande que les oscillations de vitesse angulaire soient nulles.

A ce sujet on n'a pas, d'après les connaissances des Auteurs, des essais d'interprétation théorique.

Dans le laboratoire du Centre de Recherche d'Hydraulique et de Structures de l'ENEL (à Milan) on a réalisé un circuit expérimental où l'on peut solliciter la machine soit par des pulsations de débit, en mesurant sa réponse en pression, couple et vitesse angulaire, soit par des pulsations de vitesse angulaire, en mesurant alors sa réponse en débit, pression et couple.

Les essais sont en cours : dès maintenant on peut tirer seulement quelques indications tout à fait générales, qui montrent une bonne cohérence entre les nouveaux essais et les essais de Fanelli (1972). Le comportement de la machine, du moins pour petites fréquences réduites, peut être schématisé par une simple impédance. Le fait est confirmé par l'observation que lorsque le système est sollicité par des oscillations de débit, l'analyse en fréquence des signaux de vitesse angulaire ne montre pas des composantes déterministes qui soient significatives au niveau des tests statistiques communs.

La ligne d'arbre ne répond pas, donc, par des pulsations de vitesse et la machine est donc sollicitée par un vecteur d'input $\varphi=\bar{\varphi} ; \nu=0$. Dans ce cas la réponse du système montre que le couplage entre les signaux de couple hydraulique et les signaux de débit est très faible $\left(\mu_{q} ₹ \mu_{q}^{o}\right)$ : cela va donc dans le sens des résul-

(1) Récemment Brennen a présenté (JFM 1978) une proposition d'interprétation théorique d'inducteur cavitant. On peut espérer que des analyses semblables soient proposées pour les machines à faible vitesse spécifique. 
tats déjà connus auparavant, à partir desquels on doit supposer une nette diminution de la partie résistive de l'impédance et donc, si $\nu \cong 0$, aussi $\tau \cong 0$.

\section{Conclusions}

On a essayé de donner un cadre des connaissances expérimentales qu'on a sur le comportement nonstationnaire des turbomachines hydrauliques.

En ce qui concerne le rôle "passif" des pompes en l'absence de cavitation, on peut retenir que les résultats obtenus donnent des indications d'intérêt, du moins pour machines de petite taille.

$\mathrm{Au}$ contraire sur grandes machines (et surtout pour les turbines) il apparaît nécessaire une expérimentation spécialement conçue et une réorganisation des données expérimentales déjà connues.
Il faut tirer les mêmes conclusions pour le comportement non-stationnaire en présence de cavitation et dans le domaine du rôle "actif" des machines, où les indications expérimentales ne présentent pas un caractère conclusif.

\section{Bibliographie}

FANELLI M. - Current studies on instationary behaviour of hydraulic machinery, 6th IAHR Symposium, Rome 1972.

FANELLI M. - Further considerations on the dynamic behaviour of hydraulic turbomachinery, Water Power, June 1972.

SICCARDI F. - Cavitation Control in pumps, L'Energia Elettrica, 4, 1976

NG S.L. and BRENNEN C. - Experiments on the dynamic behaviour of cavitating pumps, Trans. ASME, J. Fluids Eng., June 1978.

BRENNEN C. - Bubbly flow model for the dynamic characteristics of cavitating pumps, J. Fluid Mechanics, 89/2, 1978.

\section{Discussion}

M. le Président. - Je remercie M. FANELLI et M. SICCARDI de l'intérêt de leur présentation, et je vous propose d'ouvrir la discussion.

M. BOUSSUGES. - Pour compléter ce que viennent de dire MM. FANELLI et SICCARDI, je dois signaler qu'à Neyrpic, nous avons continué les essais que nous avions exposés trop succinctement à la dernière réunion, pour lesquels les résultats n'étaient pas très probants. Nous nous heurtons en effet à deux difficultés : d'une part, le problème de mesure des débits à fréquence relativement élevée et, d'autre part, la résonance de conduite qui était assez basse et qui masquait pratiquement les résultats.

Actuellement, nous avons réalisé de façon à peu près correcte l'essai sur une banđe de fréquence comprise entre $1 H_{z}$ et environ $15 H_{z}$ ? La mesure de débit a été faite par un débitmètre à ultrason qui a permis, après maintes difficultés, de donner des tésultats qui confirment, en gros, les calculs que nous avions exposés.

En résumé, la turbine répond comme un phénomène passif sans partie imaginaire côté fréquence de l'ordre de $1 H_{z}$ ou $1 / 2 H_{z}$ et on l'accroît de plus en plus jusqu'à $15 H_{z}$. Au-delà, nous n'avons pas pu aller sur des fréquences plus élevées par suite de difficultés que nous avons en ce qui concerne la mesure du débit.

M. HUFFENUS. - J'ai beaucoup apprécié dans l'exposé de M. FANELLI les réserves qu'il a formulétes sur le caractère inévitablement un peu aventureux de 1'approche qu'il conduit du fait de la linéarisation. On peut en effet concevoir quelques inquiétudes à ce sujet lorsqu'apparaissent au cours d'un transitoire des décollements, cavitation ou tout autre phénomène modifiant profondément le comportement de la machine. Dans cet esprit, il serait intéressant que $M$. SICCARDI nous précise l'ordre de grandeur des "petites" perturbations dont il étudie les répercussions, car pour être petites, celles-ci doivent être assez gxandes pour être appréciables si bien que les coefficients de l'impédance n'en sont plus indépendants.

M. SICCARDI. - Sur la machine de l'ENEL, les pulsations de débit sont de $5 \%$ du débit moyen. Sur la machine de l'Ins- titut d'Hydraulique de Gênes les pulsations sont de l'ordre de $10 \%$ du débit moyen. Sur le petit inducteur d'Agosta-Brennen, j'ai pu tirer des données expérimentales, qui ont été publiées, pour des pulsations de l'ordre de 2 à $5 \%$ du débit moyen. Je pense que la linéarisation est légitime.

M. CASACCI. - Vous utilisez une matrice carrée du $2^{\mathrm{e}}$ ordre ou une matrice du $3^{\mathrm{e}}$ ordre, quels phénomènes représentent cette dernière?

M. FANELLI. - Il y a des effets de compressibilité du fluide dus à la cavitation.

M. CASACCI. - Je pense que l'étude d'une matrice carrée $\mathrm{du} 2^{\mathrm{e}}$ ordre constitue déjà un problème très délicat et qu'il vaudrait mieux se limiter à son étude.

Si l'on admet la linéarité des phénomènes, on peut déjà obtenir une notion des fonctions de transfert en jeu en introduisant des signaux aléatoires. N'y aurait-il pas là une voie d'étude possible ? Produire une variation de débit sinusoïdale reste très difficile, ne vaudrait-il pas mieux injecter une entrée quelconque bien définie et mesurer la réponse du système ?

M. FANELLI. - En ce qui concerne la première question, en effet, pour le moment, nous nous bornons au cas du quadriterme, c'est-à-dire la matrice $2 \times 2$, et nous contrôlons les variables, soit les variations périodiques de débit, soit les variations périodiques de vitesse angulaire. Les résultats qui ont été présentés se rapportent notamment aux variations périodiques de débit à vitesse angulaire constante. Mais une deuxième série que nous espérons mener à bien cette année se rapporte à des variations de vitesse angulaire contrôlée. Ceci veut dire que nous serons à même, si les mesures sont correctes, de caractériser le quadriterme. Je crois que ceci répond à votre première question.

En ce qui concerne la deuxième question, en effet, en principe il est possible d'utiliser des perturbations quelconques, d'autant plus que nous sommes obligés de mettre en ceuvre des moyens d'élaboration et de traitement des signaux qui sont déjà sophistiqués, même quand nous injectons une perturbation sinusoïdale. Mais l'approche qui emploie une perturba- 
tion sinusoïdale dérive plutôt de raisons historiques. On commencé à une époque où l'on ne disposait pas de moyens de traitement très élaborés et on préférait connaître un signal à une certaine fréquence bien définie, car on pouvait voir ce que le système donnait.

Nous avons poursuivi dans cette voie, d'autant plus qu'il n'est pas difficile d'injecter, non pas dans la machine, mais dans le système, une perturbation de débit très pure; on y est parvenu correctement. C'est autre chose pour des perturbations d'autre nature, notamment pour des vitesses angulaires ou des pressions ; mais, pour les débits, c'est très possible.

On a trouvé commode d'utiliser l'installation mise au poin pour injecter des variations sinusoïdales de débit. Mais je suis d'accord avec vous : il est possible, et même prometteur, d'utiliser un signal aléatoire quelconque par exemple pour les relevé que l'on fait en vraie grandeur ou sur des modèles de machines. J'espère que les constructeurs pourront retenir cette suggestion.

M. CASACCI. - Industriellement on peut mesurer par exemple, pendant un certain temps, la variation de vitesse, la variation đe débit, la variation de chute à l'entrée et à la sortie. On devrait pouvoir tirer des informations de ces mesures industrielles qui viendraient confirmer les recherches sur modèle réduit.

M. LOURME. - Nous travaillons à l'ONERA sur les problèmes d'hydraulique instationnaire, en particulier pour caractériser les systèmes d'alimentation du lanceur Ariane en vue d'analyser sa stabilité pogo. Nous disposons à Palaiseau d'un banc d'essai qui nous a permis de déterminer les matrices de transfert d'éléments simples tels que canalisations droites, diaphragmes, accumulateurs... ou plus complexes tels que des boitiers de régulation ; pour la mesure des débits instationnaires, nous avons développé un débit-mètre à ultrasons (commercialisé depuis par Crouzet) qui donne entière satisfaction (la NASA utilise d'ailleurs ces débitmètres pour la Navette Spatiale). En ce qui concerne les pompes, nous n'avons pas encore nous-mêmes fait d'essais, mais nous avons développé à partir d'essais effectués par la SEP un modèle théorique analogue à celui de Brennen.

Je voudrais main tenant vous poser deux questions:

1) quand la matrice de transfert se réduit à une impédance, avez-vous cherché à retrouver par la théorie le terme imaginaire observé expérimentalement ?
2) quand la matrice de transfert est un quadripôle, vous avez besoin de 2 essais distincts pour déterminer les 4 termes, sauf si vous utilisez comme Brennen 2 excitations simultanées : comment avez-vous procédé ?

M. FANELLI. - En ce qui concerne la première question, je dirai qu'on avait, autrefois, tenté de relier les variations du terme imaginaire de l'impédance à un traitement théorique plus ou moins discutable ; on entrevoyait quelque chose, mais c'était trop vague pour être vraiment convaincant.

La deuxième question posée concernait la détermination des éléments du quadriterme. Nous avons pensé opérer de la façon suivante : dans une série d'essais, nous injectons des variations de débit et la vitesse angulaire est pratiquement constante. Dans l'autre série d'essais, nous injectons des variations contrôlées de vitesse angulaire qui entraînent à leur tour des variations de débit qui peuvent être de quelque manière mesurées. Dans cette deuxième série d'essais, on a deux variations de grandeur d'entrée, mais dans des conditions nettement différentes de la première série d'essais ; donc, en principe, on peut déterminer deux éléments différents.

M. LOURME. - Vous avez une seule source de fluctuations ?

M. FANELLI. - Les points d'application sont différents. Des débits oscillants sont in troduits pour déterminer le circuit, dans notre cas, à l'aval de la pompe, et les variations de vitesse angulaire sont introduites à l'amont.

M. le Président. - Je voudrais moi-même formuler une remarque. Dans ce que vous nous avez exposé, vous semblez lier de manière très nette la cavitation à l'apparition d'un terme capacitif dans la partie imaginaire de l'impédance. Sait-on pourquoi ? Est-ce que le fait d'introduire un terme capacitif, donc la possibilité d'excitations entretenues, est lié au phénomène de cavitation et au fait qu'on peut avoir extraction d'énergie dans le circuit pour la libérer ensuite ?

Je n'ai pas très bien vu pourquoi la cavitation était liée à l'apparition d'un terme capacitif.

M. SICCARDI. - Les résultats de Brennen en présence de cavitation montrent bien l'apparition, à travers la machine, non seulement d'un terme capacitif mais aussi d'un terme d'amplification. Lorsque la machine cavite elle présente un comportement complet de quadripole. 